\title{
The effects of the tylosin and Cd on soil enzyme activity
}

\author{
Zhaohong Meng ${ }^{1}$, Shuman Wang ${ }^{1}$, Jia Zhou ${ }^{2 *}$ \\ ${ }^{1}$ Department of City and Tourism, Luoyang Normal University, Luoyang 471022, China \\ ${ }^{2}$ College of Geographical Sciences, Harbin Normal University, Harbin150025, P. R. China
}

\begin{abstract}
Soil microbial environment have been affected by different concentration heavy metals Cd (HM) and tylosin (TYL) and combination of TYL and HM interactions. Degradation of TYL was caused certain inhibition due to the addition of HM. The germination index of seed had been inhibited owing to the toxic effects of HM and TYL, but we found that the low concentrations of HM (4 mg/kg), the germination index higher than the soil which unadded HM and TYL in it. The soil enzyme activity was significantly suppressed by the addition of HM and TYL. Actinomycete was inhibited by high concentrations of HM for a long time. The studies demonstrated that the pollution of the soil micro-environment has been serious than only add HM or TYL in the soil.
\end{abstract}

\section{Introduction}

In recent years, the acceleration of industrial development, industrial waste pollution, fertilizers and pesticides, and mining have accelerated the pollution of heavy metals by soil[1]. In last health of livestock and human were affected that because most of heavy metals were steadily in soil, through the food chain[2]. There were exceed 20 million ha of land were polluted by heavy metals like $\mathrm{As}, \mathrm{Cd}, \mathrm{Cr}, \mathrm{Cu}, \mathrm{Ni}, \mathrm{Zn}$, and $\mathrm{Se}$ in globally, which higher than the baseline or regulatory levels[3] Since most of the heavy metals Cd were not absorbed by livestock, most of the heavy metals $\mathrm{Cd}$ are expelled directly from the body. Wang C X et al.[4] studied heavy metals $\mathrm{Cd}$ causes oxidative stress, chlorophyll, and synthesis of sugar and protein. The feed additives contain antibiotics and heavy metals were recognized in many country. Heavy metals and antibiotics are often added at the same time in livestock breeding. Moreover, adding two kinds of interference at the same time may produce more intense toxicity. Carla Vignaroli et al.[5] studied showed the selection of antibiotic-resistant enterococci was stimulated by any concentration of heavy metal. István Máthé et al.[6] studied indicated the presence of heavy metal had accelerated the production of an antibiotic resistant gene. $\mathrm{Yu}$ Zhang et al.[7] studied showed compound pollutants produced by the combination of heavy metals and antibiotics are more toxic. However, reraly studies were considered to make a thorough inquiry the single and overall inhibitory effects of heavy metal $\mathrm{Cd}(\mathrm{HM})$ and tylosin (TYL) in soil microbial environment. To clarify the inter-influence of heavy metal $\mathrm{Cd}(\mathrm{HM})$ and tylosin (TYL) on soil to obtain information soil microbial environment change. The purpose of this paper is to discuss the effects of TYL and HM on soil microbial environment.

\section{Materials and Methods}

\subsection{Sample collection and analyses}

The soil is mainly collected and the upper part of the selected area is $0-20 \mathrm{~cm}$ at Harbin suburbs, Heilong Jiang Province, China. The soil contained $1.79 \%$ organic carbon, the water holding capacity was $3 \%$ and $\mathrm{pH}$ was 7.53 (Heidmann, 1989). The soil sample was brought to the laboratory and stored at $4^{\circ} \mathrm{C}$ in the dark. The heavy metal elements were determined by an ICP-MS (7700x series, Agilent Technologies, Santa Monica CA, USA) equipped with an octopole reaction system.

The soil was mixed, sieved $(2 \mathrm{~mm})$, air dried overnight at $28-30^{\circ} \mathrm{C}$ a week and transferred to nine flower pot (1000g soil in each). The specifications of the flower pot are $150 \mathrm{~mm} \times 150 \mathrm{~mm} \times 200 \mathrm{~mm}$ (bottom width $\times$ multiplied $\times$ height). TYL treated soils were prepared by first dissolving TYL (tylosin tartrate, Sigma) and $\mathrm{Cd}\left(\mathrm{CdCl}_{2}, 99 \%\right.$ analytical purity, Tian Jin) in water and then thoroughly mixing the solution into the soil to a final concentration of 500 and $2000 \mu \mathrm{g} \mathrm{TYL} \mathrm{g}^{-1}$ dry soil concentration of 0.4 and $1.6 \mu \mathrm{g} \mathrm{CdCl}_{2} \mathrm{~g}^{-1}$ and a water content of $15 \%$ corresponding to the water holding capacity of the soil, once every two days to adjust to keep the moisture content. TYL concentration gradient is $500 \mathrm{mg} / \mathrm{kg}, 2000 \mathrm{mg} / \mathrm{kg}$, the HM concentration gradient is $4 \mathrm{mg} / \mathrm{kg}, 16 \mathrm{mg} / \mathrm{kg}$. The CK is the control check; The TYL-L joined TYL $500 \mathrm{mg}$ in; TYL-H joined $2000 \mathrm{mg}$ TYL; HM-L adding the $\mathrm{CdCl}_{2}$ of $4 \mathrm{mg}$; HM-H adding the $\mathrm{CdCl}_{2}$ 16mg; TYL-L+HM-L the TYL $500 \mathrm{mg}$ joined and $4 \mathrm{mg} \mathrm{CdCl}$; TYL-L+HM-H, TYL joined $500 \mathrm{mg}$ and $16 \mathrm{mg} \mathrm{CdCl}$; TYL-H+HM-L, TYL joined $2000 \mathrm{mg}$

* E-mail: harbin_zhoujia@hrbnu.edu.cn 
and $4 \mathrm{mg} \mathrm{CdCl} 2$; TYL-H+HM-H, TYL joined $2000 \mathrm{mg}$ and $16 \mathrm{mg} \mathrm{CdCl}$. Three parallel controls for each group of test samples. The soil was incubated aerobically at $30^{\circ} \mathrm{C}$ in the dark, and the water content was held constant. On day $1,2,4,7,15,30,50$ were obtained from soil samples $(100 \mathrm{~g})$ at 4 o'clock afternoon, soil respiration, alkaline phosphatase, catalase, urease, invertase, TYL residues, fungi, bacteria and actinomycetes. At $50^{\text {th }}$ day stop experiment record.

\subsection{Degradation of TYL}

Accurately weighing $2.000 \mathrm{~g}$ samples fresh soil, adding $10 \mathrm{ml}$ extractant (acetonitrile: water $=9: 1$ ) into $50 \mathrm{ml}$ centrifuge tube, vortex $1 \mathrm{~min}$, ultrasonic $30 \mathrm{~min}, 4000$ rpm centrifugation $5 \mathrm{~min}$, retain its supernatant, the above operation is carried out two times. At $40^{\circ} \mathrm{C}$, rotating and evaporating, adding $0.5 \mathrm{ml}$ methanol, $4.5 \mathrm{ml}$ $0.15 \mathrm{~mol} / \mathrm{L}$ sodium acetate $(\mathrm{pH}=5.5)$ and vortex $1 \mathrm{~min}$, transferred to $10 \mathrm{ml}$ centrifuge tube. First with $5 \mathrm{~mL}$ methanol, $5 \mathrm{ml}$ ultra-pure water activated SPE column, then add the $5 \mathrm{ml}$ sample after enrichment with eluent, elution (2\% ammonia water, 5\% methanol, 93\% water), eluting with $5 \mathrm{ml}$ methanol, liquid phase filter membrane into the vial to be measured. Determination of TYL Residues Using HPLC (Hatachi, Primaide, UV: 190600nm, DAD: 190-900 nm). Chromatographic conditions: Agilent TC-C18 column $(4.6 \mathrm{~mm} \times 250 \mathrm{~mm}$, $5 \mu \mathrm{m})$, mobile phase: acetonitrile $0.01 \mathrm{~mol} / \mathrm{L}$ potassium dihydrogen phosphate solution $(\mathrm{pH}=2.5)$ gradient elution, elution conditions: Elution procedure: 0-18 min elution with acetonitrile, potassium dihydrogen phosphate solution at 20: 80 volume ratio. Flow rate: $1 \mathrm{ml} / \mathrm{min}$, detection wavelength: $290 \mathrm{~nm}$, injection volume: $20 \mu \mathrm{l}$, column temperature: $30^{\circ} \mathrm{C}$. Detection limits: $0.2 \mu \mathrm{g} / \mathrm{kg}$. Recovery rate: $92.35 \sim 101.23 \%$. As a result of the TYL Easy to degrade, this condition can be used to detect TYL residues.

\subsection{Soil enzyme activity assays}

Urease (EC 3.5.1.5) activity was measured according to Tabatabai (1982). Alkaline phosphatase (EC 3.1.3.1) activity was measured according to the method described by NT Davies (1978). Invertase (EC 3.2.1.26) analysis was conducted according to the method of Ross (1975). All enzyme assays were conducted with toluene, added $15 \mathrm{~min}$ before the addition of buffer and substrate. Enzyme activities were expressed on a soil dry weight basis.

\section{Test Results and Discussions}

\subsection{Tylosin of degradation in soil}

The degradation behaviors of TYL at different concentrations in soil were shown in Fig.1.and Fig.2. The concentrations of TYL were 1.087(TYL-L), 5.4781(TYL-H), 0.3569(TYL-L+HM-L), 12.5365(TYLL+HM-H), 0.5874 (TYL-H+HM-L) and 5.6873(TYL$\mathrm{H}+\mathrm{HM}-\mathrm{H}) \mathrm{mg} / \mathrm{kg}$, with the degradation ratios of 99.72 , $99.78,99.37,99.92,99.55$ and $99.88 \%$ in the soil, respectively. The degrade number of the TYL can expressed and coincided with the kinetic models. The results showed degrading TYL was consistent with the first-order reaction kinetics model. The first-order kinetic constants (k) for degradation were 0.1233(TYL-L, $\left.\mathrm{R}^{2}=0.9967\right), 0.1016\left(\mathrm{TYL}-\mathrm{H}, \mathrm{R}^{2}=0.9987\right), 0.1011$ (TYL$\left.\mathrm{L}+\mathrm{HM}-\mathrm{L}, \quad \mathrm{R}^{2}=0.9974\right), \quad 0.1016(\mathrm{TYL}-\mathrm{L}+\mathrm{HM}-\mathrm{H}$, $\left.\mathrm{R}^{2}=0.9987\right), \quad 0.0981\left(\mathrm{TYL}-\mathrm{H}+\mathrm{HM}-\mathrm{L}, \quad \mathrm{R}^{2}=0.9993\right)$ and 0.0967 day $^{-1}\left(\mathrm{TYL}-\mathrm{H}+\mathrm{HM}-\mathrm{H}, \mathrm{R}^{2}=0.9996\right)$ respectively. The degradation half-lives $\left(\mathrm{t}_{1 / 2}\right)$ of TYL-H (5.61 days) was the highest, the treatment is TYL-L+HM-H (7.15 days) was the lowest. TYL degraded fast in days 0-6. For treatments TYL-H, TYL-H, TYL-L+HM-L, TYLL+HM-H, TYL-H+HM-L and TYL-H+HM-H, $12.03 \%$, $11.97 \%, 11.73 \%, 11.79 \%, 11.43 \%$ and $11.72 \%$ TYL were degraded average a day, respectively. In the 15th, the TYL degradation rates were $90.87 \%, 92.38 \%$, $90.03 \%, 91.64 \%, 87.65 \%$ and $91.54 \%$ for treatments TYL-H, TYL-H, TYL-L+HM-L, TYL-L+HM-H, TYL$\mathrm{H}+\mathrm{HM}-\mathrm{L}$ and TYL-H+HM-H, respectively. The results suggested that TYL degradation was inhibited by HM. There was significant difference in TYL degradation in all the treatments according to the one-way ANOVA analysis $(P<0.05)$.

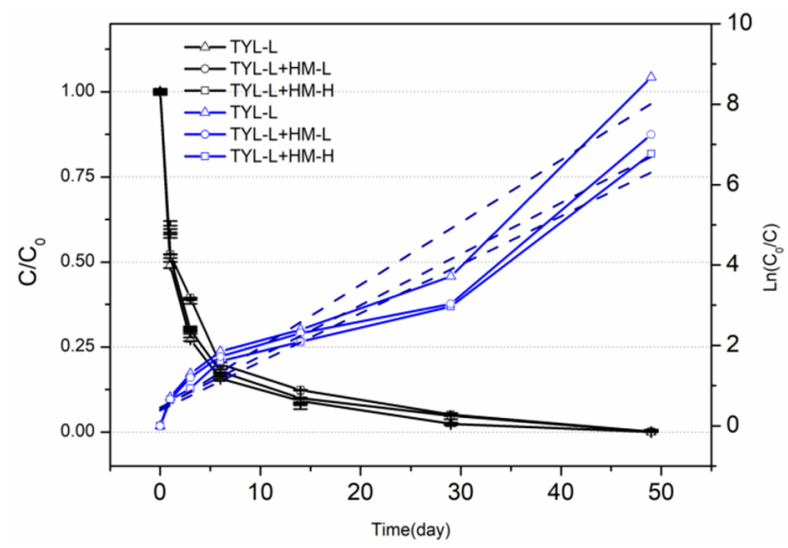

Fig.1 Percentage of degradation and degradation rate of TYL-L=500mg $/ \mathrm{kg}$ at different concentrations. 


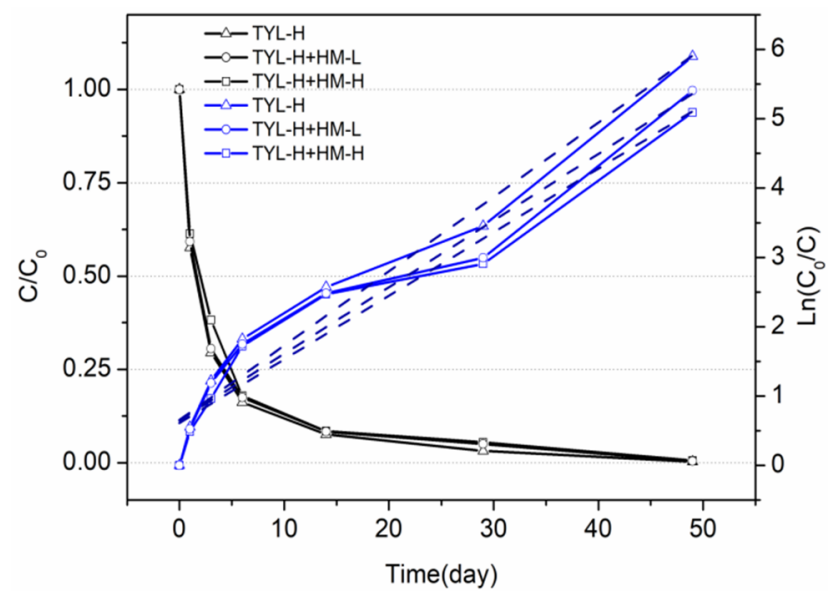

Fig.2Percentage of degradation and degradation rate of TYL-H=2000mg $/ \mathrm{kg}$ at different concentrations.

Build-up of HM will sure to happen destroy natural soil microflora and soil-enzyme, and lower soil quality. The degradation half-life of TYL (TYL-L) in treatment TYL-L (5.61 days) soil was the highest. The treatment TYL-L+HM-H (7.06 days) soil was the lowest. The degradation half-life of TYL (TYL-H) in treatment TYL$\mathrm{H}$ (6.01 days) soil was the highest, the treatment TYL$\mathrm{H}+\mathrm{HM}-\mathrm{H}$ (7.15 days) soil was the lowest. Degradation rate of TYL in soil and the number of HM was to some extent the trend of negative correlation. We believe degrading TYL was inhibited by high concentrations of HM. The HM exists in the form of HM-ion in the soil and with it microbial restraint of indirect effected in degradation of TYL. And the toxic succeed of heavy metal will also kill a part of microbes. This showed the HM might present toxicity effects on soil microbial. The number of microorganisms became bulk and bulk was caused by HM, because of it changed the degradation rate of TYL. The results showed the TYL degradation are well described a first-order reaction kinetics model. The values of the clear rate constant $\mathrm{k}$ presented the opposite evolution than the degradation efficiency. They increased with decreased HM concentration. The values of the plain rate constant $\mathrm{k}$ can show the speed of degradation of TYL. In summary, we have identified the degrading of TYL was received reserve by the increase of concentrating of HM.

\subsection{Soil enzyme activity}

\subsubsection{Soil urease activity}

Soil urease concentration trend was showed in Fig.3. Urease is a foundation the index of Biological and fertility status by soil. In the entire of Simulation experiment, the urease decrease in the first seven days and then increased. In the early stage of composting, urease for all treatments were lower than in the control, in the TYL-H+HM-H simulation experiment was $24.62 \%$ lower than that in the control. Then, the increased significantly with increasing time $\left(30^{\text {th }}\right.$ day). In this period, the urease of the treatments (HMH, TYL-L+HM$\mathrm{H}$ and TYL-H+HM-H) was obviously slower than those of other samples. The experiment reflects the amount of urease was higher than the CK in the sample with low HM content. This shows that urease at low concentrations of HM was not toxic, but instead stimulates, hastening the role and amount of catalysis. In treats high concentration HM samples, the urease and concentrate metal showed a reversed.

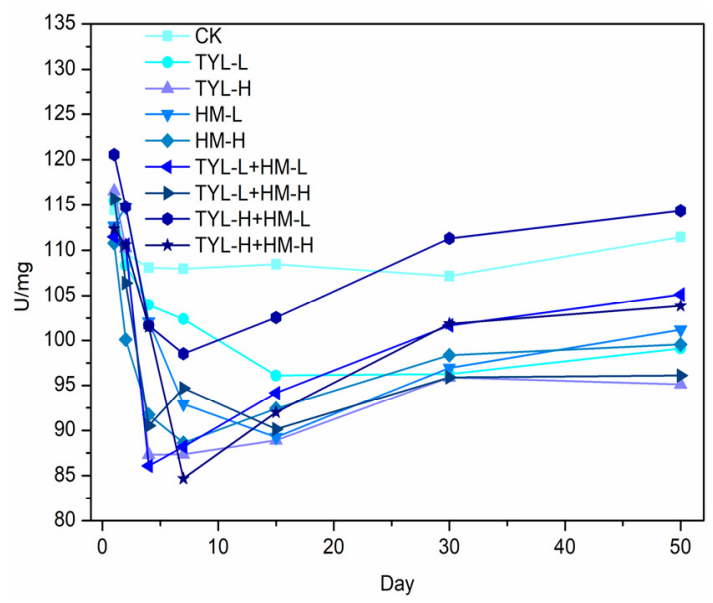

Fig 3. Soil urease concentration trend

\subsubsection{Soil alkaline phosphatase activity}

Soil alkaline phosphatase concentration trend was showed in Fig.4. The alkaline phosphatase activity is an originally the index of humus and organo-phosphorus 
compounds from soil. In a word, alkaline phosphatase activity reflected in soil fertility and phosphate fertilizer. In the early experiment $\left(4^{\text {th }}\right.$ day), the alkaline phosphatase activities for all treatments were relatively lower than their counterparts in the later stage. At the beginning of the experiment, the alkaline phosphatase activities were suppressed by TYL and HM. During the experiment (HM-L and TYL-L+HM-L) were obviously higher than those of other samples, with $8.48 \%, 3.49 \%$ increases compared with those in the CK on $50^{\text {th }}$ days, respectively. Moreover, the alkaline phosphatase activity was very sensitive to high-HM and high-TYL(TYL-H, HM-H, TYL-H+HM-L and TYL-H+HM-H), which decreased $18.78 \%, \quad 16.17 \%, 22.19 \%$ and $23.29 \%$ contrasted with the $\mathrm{CK}$ on the $50^{\text {th }}$ days. Alkaline phosphatase showed a negative correlation trend with high concentration of TYL and high concentration of HM.

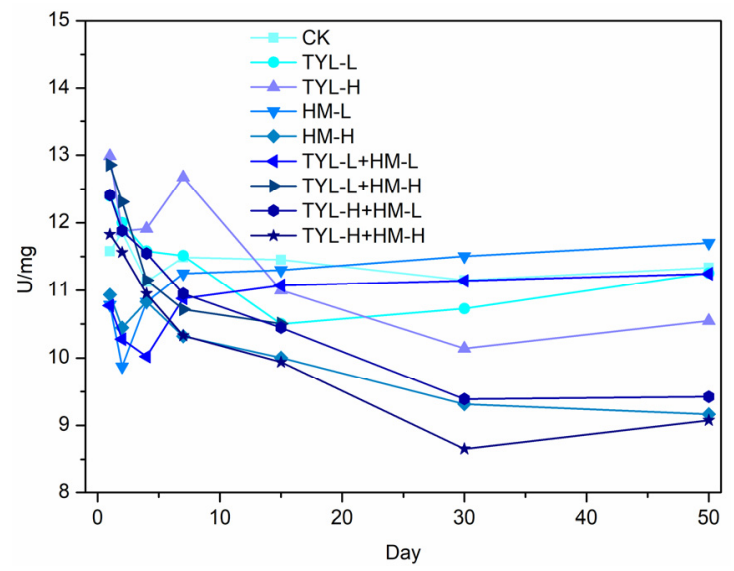

Fig 4. Soil alkaline phosphatase concentration trend

\subsubsection{Soil Invertase activity}

Soil invertase concentration trend was showed in Fig.5. Invertase is able to catalyze sucrose and raffinose hydrolysis to be transformed into glucose fructose and two-sugar, which are closely related to the microbial quantity and respiratory intensity in soil (Zhang et al., 2018). Compared with other enzyme activities, invertase activity was not significant in the group, but the difference between the groups was obvious. In the entire process of Simulation experiment, the invertase activities generally tended to decrease in the $15^{\text {th }}$ days and then increased thereafter. At the first day, activity in the all treatments simulation experiment was 42.64-83.84\% lower than that in the CK. Although at the finish of the experiment, the invertase trend to increased, but the treatments compared to $\mathrm{CK}$ was suffered great influence, decreases of $60.56-78.54 \%$ in the invertase activity was detected compared with the activities in the $\mathrm{CK}$ at $50^{\text {th }}$ days, respectively. The result showed that the invertase were inhibited by TYL and HM.

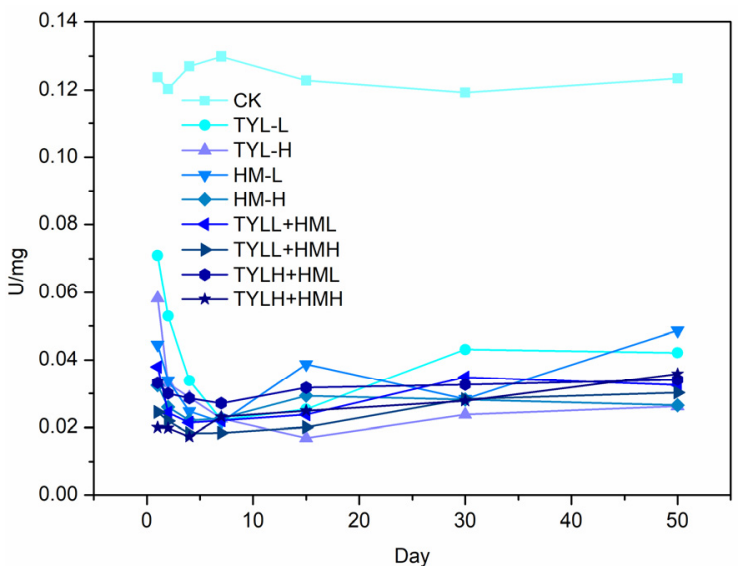

Fig 5. Soil invertase concentration trend

Decomposition of organic matter and detoxification of aerobic compounds are carried out by enzymatic activity, since enzyme activity plays a mediating role in biogeochemical processes. So they can be potentially used as a signature of the mechanism and rate of biotransformation as well as the functionality of the ecosystem. But the presence of toxic compounds, HM,
TYL, for instance, can obstruct (activate or inhibit) the enzymatic activity and subsequently the magnitude of ecosystem metabolism and pollutants alteration. This result indicated that in the process of simulation experiment. HM and TYL presents intensified inhibitory effects as the content increased. This inhibitory effect possibly arose not only because the HM presented a poisoning effect on the specific enzyme, but also because 
of a concomitant TYL had a killing effect on microorganisms. On the other hand, HM was stably deposited in the soil. However, the enzyme activity shows a trend of recovery after a period of time. Continuous degradation of TYL inhibition weakens gradually by the time. Enzyme activities were stimulated microorganisms to produce by TYL. Another possible interpretation that this effect was due to the development of microbial tolerance to the pressure posed by toxic ants during the simulation experiment process $(\mathrm{P}<0.05)$.

\section{Conclusion}

The present paper proves the inhibition of the combine effected TYL and HM in soil. The degradation rates of TYL in this study fit a first-order kinetic model, and the degradation half-life of TYL was lowest in treatments (TYL-L+HM-H and TYL-H+HM-H), followed by treatments (TYL-L+HM-L and TYL-H+HM-L) and treatments (TYL-L and TYL-H). Under the influence of HM, the degradation rate of TYL was inhibited. The soilmicrobes were inhibited by the presence of HM. The soil microbial activity was significantly inhibited with the increase of the concentration of HM. Soil microbial activity was an important indicator of the quality of soil in response to soil. Further microbial activity through soil enzyme activity to express. The presence of HM resulted in enzyme activity, which could partially explain the lower TYL degradation rate in the treatments. In the micro-environment of soil, the inhibition of high concentration of heavy metals is higher than that of TYL, however the low concentration of HM stimulates the activity of microorganisms under comparable conditions. With the addition of HM, it will adsorb soil microbes and antibiotics, which will also inhibit the role of TYL in the soil. TYL adsorption on soil minerals was a spontaneous process. In the TYL and combined effects of HM, and more likely to enhance the occurrence of joint toxicity. Due to TYL was easy to degraded, it had little effected on HM. The effect of highly toxic HM on soil microenvironment was stable for a long period.

\section{Acknowledgments}

This work has been financially supported by the Nature Science Foundation of Heilongjiang Province (No. D2018004) and (No. D2018002). The higher Education Teaching Reform Research and Practice Project of Henan Provincial Department of Education(No. 2019SJGLX390). Youth Backbone Teachers Project of Henan Colleges and Universities(No. 2018GGJS130). Luoyang Normal University education reform project(No. 2019XJGJ034).

\section{References}

1. Fang FM., Wang Q., Research progress on mercury pollution in soil. Soil Environ; 2000, 9: 326-329.

2. Dar G. H., Mishra M. M., Influence of cadmium on carbon and nitrogen mineralization in sewage- sludge amended soils. Environ. Pollut. 1994, 84: 285-290.

3. Lianwen Liu., Wei Li., Weiping Song., MingxinGuo., Remediation techniques for heavy metal-contaminated soils: Principles and applicability. Sci. Total Environ. 2018,6: 206-219

4. C.X Wang., Z Mo., H Wang., Z J Wang., Z H Cao., The transportation, time-dependent distribution of heavy metals in paddy crops. Chemosphere. 2003,50: 717-723.

5. Carla Vignaroli., Sonia Pasquaroli., Barbara Citterio., Andrea Di Cesare., Gianmarco Mangiaterra., Daniele Fattorini., Francesca Biavasco,. Antibiotic and heavy metal resistance in enterococci from coastal marine sediment. Environ. Pollut. Vol. 2018, 237: 406-413.

6. István Máthé., Tibor Benedek., András Táncsics., Márton Palatinszky., Szabolcs Lányi ., Károly Márialigeti., Diversity, activity, antibiotic and heavy metal resistance of bacteria from petroleum hydrocarbon contaminated soils located in Harghita County (Romania). Int biodeter biodegr. Vol. 2012, 73: 41-49.

7. Yu Zhang., Xiyun Cai., Xianming Lang., Xianliang Qiao., Xuehua Li., Jingwen Chen., Insights into aquatic toxicities of the antibiotics oxytetracycline and ciprofloxacin in the presence of metal: Complexation versus mixture. Environ. Pollut. Vol. 2012,166: 48-56. 